\title{
Shuttlebox avoidance to intense white noise: Acquisition and the Kamin effect in rats
}

\author{
RICHARD A. HUGHES and C. WILLIAM BRETT \\ Virginia Polytechnic Institute and State University, Blacksburg, Virginia 24061
}

\begin{abstract}
Experiment I demonstrated shuttlebox avoidance conditioning using intense white noise as a UCS. Ten rats were given 25 trials a day for 6 days. Escape latencies declined and avoidance responses increased over trial blocks. Experiment II provided support for a functional similarity between shock as a UCS and intense noise as a UCS by demonstrating the Kamin effect following incomplete shuttlebox training to noise. Separate groups of rats were given 25 trials followed by an additional 25 trials either 0 , 1,4 , or $24 \mathrm{~h}$ later. The U-shaped Kamin effect was evident in the avoidance measure. A similar but

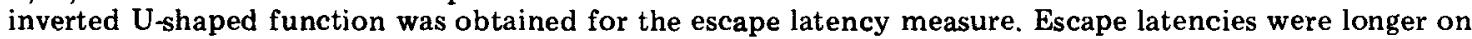
retraining than on original training at $1 \mathrm{~h}$ but not at 0,4 , or $24 \mathrm{~h}$ after original training.
\end{abstract}

Intense noise has been used successfully to condition relatively simple escape and avoidance responses (Barnes \& Kish, 1957; Campell, 1955), but attempts to condition more complex responses such as barpress escape or avoidance have been less successful (Barry \& Harrison, 1957; Harrison \& Abelson, 1959; Harrison \& Tracy, 1955). The relatively modest degree of conditioning that has been reported in some situations may be a result of insufficient motivation provided by the noise stimulus (Campbell \& Bloom, 1965), or to noise-elicited responses that are incompatible with the response to be conditioned (Bolles \& Seelbach, 1964). This latter interpretation is similar to and consistent with Bolles (1971) analysis of the relationship between species-specific defense reactions (SSDRS) to shock and ease of conditioning. A functional similarity between noise and shock is evident in the fact that relatively simple escape and avoidance responses to shock, as is the case with noise, are rapidly conditioned, and more complex responses to shock, such as barpress escape or avoidance, as is the case with noise, are much more difficult to condition. On the other hand, shuttlebox avoidance to shock is more difficult to condition than escape or one-way avoidance and less difficult to condition than barpress escape or avoidance (Bolles, 1971). In this sense, shuttlebox avoidance appears to be of intermediate difficulty between escape and one-way avoidance conditioning and barpress conditioning. Shuttlebox avoidance using noise as a UCS has not been reported, but if the similarity in the ease with which other responses to noise and to shock can be conditioned is more than coincidental, then shuttlebox avoidance using intense noise should be possible.

The first purpose of the research to be reported was to examine shuttlebox avoidance in rats using intense

Supported, in part, by a grant from the Research Division, Virginia Polytechnic Institute and State University. Requests for reprints should be sent to Richard A. Hughes, Department of Psychology, Iowa State University, Ames, lowa 50011. noise as a UCS. If intense noise is sufficiently and consistently aversive to motivate acquisition of shuttlebox avoidance, then it also may be possible to demonstrate other shuttlebox avoidance phenomena with white noise. For example, Kamin (1957) reported that acquisition of an incompletely learned avoidance response to shock progresses more slowly at intermediate than at either brief or prolonged intervals after original training. Although this time-dependent U-shaped function (the Kamin effect) has been demonstrated a substantial number of times under a variety of conditions, the vast majority of replications has used shock as the UCS (Brush, 1971). Thus, the second purpose of this research was to examine the Kamin effect with intense noise.

\section{EXPERIMENT I}

\section{Method}

Subjects. Ten experimentally naive, 80-83-day-old male hooded rats, obtained from the departmental colony (derived from breeding stock obtained from Canadian Breeding Labs, Quebec) were housed individually under 24-h illumination and were maintained on ad-lib food and water.

Apparatus. Two automated shuttleboxes were used. Each shuttlebox was separated into two compartments $(19 \times 12 \times 15 \mathrm{~cm}$ each) by a guillotine door (Hunter model 194) which remained open throughout the experiment. The compartments were constructed of clear Plexiglas sides, a stainless steel grid floor $(.64 \mathrm{~cm}$ diam, separated by $2 \mathrm{~cm}$ center to center), and a hinged clear Plexiglas top. White noise (110 dB), from a Grason-Stadler noise generator (model 901B) could be delivered to speakers $(.5 \mathrm{~W} 8 \mathrm{ohm})$ that were centered over a $2-\mathrm{cm}$ hole on the opaque white Plexiglas end panel of each compartment. Noise intensity was measured by a Realistic sound level meter (model 33-1028 re., SPL $.0002 \mathrm{dynes} / \mathrm{cm}^{2}$ ). The intensity measure was determined inside the apparatus $6 \mathrm{~cm}$ from each speaker placement. A 7.5-W white light bulb extended through the ceiling at the end of each compartment. Responses were monitored by contact relays connected to alternate grids in the end of each compartment (beginning about $15 \mathrm{~cm}$ from the midpoint of the apparatus). Standard digital counters, relay circuitry, and timers were used to program all stimulus contingencies and to record escape responses, avoidance 


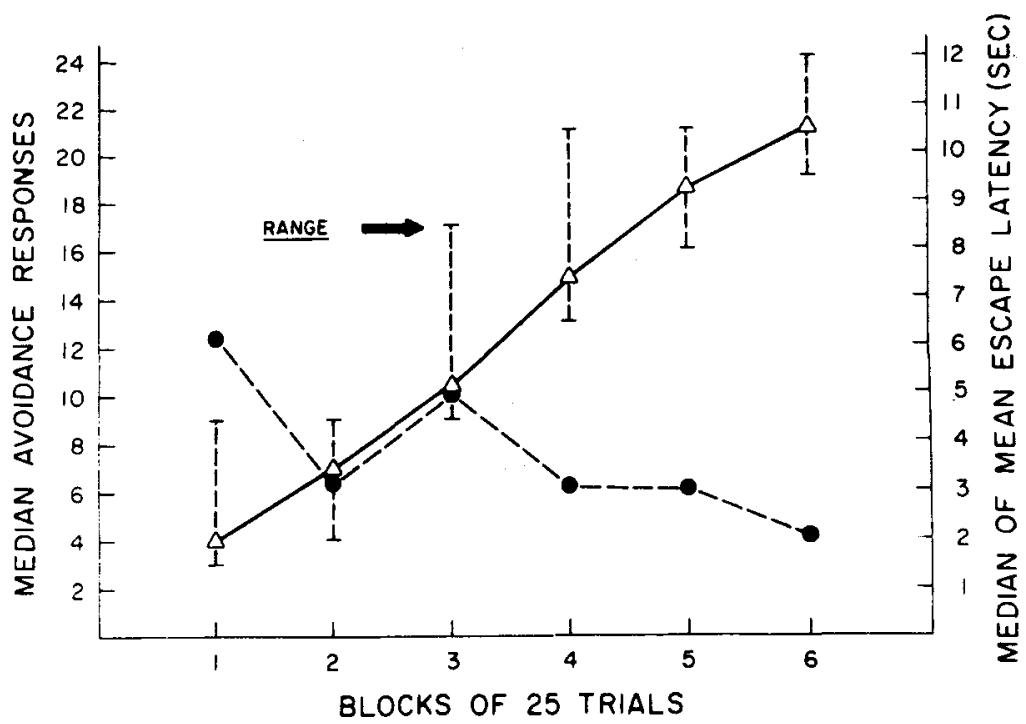

Figure 1. Median avoidance responses and median of mean escape latencies for blocks of 25 trials. The avoidance measure is represented by open triangles and solid lines, and the escape measure is represented by solid circles and broken lines.

responses, and escape latencies. The shuttleboxes were housed in sound-attenuated chambers located in a dark room adjacent to the programming and response recording equipment.

Procedure. Each animal was given 25 trials per day for 6 days. On the 1st day of training, the animal was placed into the apparatus and allowed a 2-min exploration period during which neither light (CS) nor noise (UCS) was presented. At the end of the exploration period, the training program started with the onset of the CS in the compartment occupied by the rat. A shuttle response, activating the contact relay in the opposite compartment within $10 \mathrm{sec}$ of CS onset, tumed off the CS and prevented UCS onset (avoidance response). Failure to perform an avoidance response resulted in presentation of the UCS which, with the CS, could be terminated by an escape response. Performance of either an avoidance or an escape response initiated a 35-sec intertrial interval after which the CS appeared in the compartment occupied by the rat, and the response contingencies described above were again in effect. Shuttle responses that occurred before the first UCS presentation were not recorded on the first training session but were recorded on subsequent sessions.

\section{Results}

The results of this experiment are summarized in Figure 1. The data clearly demonstrate acquisition of a shuttlebox avoidance response to intense noise. Acquisition of the shuttle response is evident as a gradual but consistent increase in number of avoidance responses and a decrease in escape latencies across trial blocks. Nonparametric statistical analysis of these data (Siegel, 1956) supported the trends evident in Figure 1. Friedman two-way analysis of variance revealed a significant increase in avoidance responses $(p<.001)$ and a significant decrease in escape latencies $(\mathrm{p}<.01)$.

The results of Experiment I are consistent with previous reports on acquisition of shuttlebox avoidance to shock (Bolles, 1971). Acquisition of the response to noise emerged gradually, requiring 150 trials to reach a level of about $84 \%$ avoidance responses.

\section{EXPERIMENT II}

Acquisition of the shuttlebox avoidance response to intense noise provided the basis for Experiment II which examined retention of an incompletely learned avoidance response in an attempt to determine the Kamin effect.

\section{Method}

Subjects. The subjects were 32 naive male hooded rats obtained from the department colony. They were about 80 days old and were maintained as described in Experiment I.

Apparatus and procedure. The apparatus was that described in Experiment I. Four groups of rats ( $\mathrm{N}=8$ per group) were given 25 training trials (original training) using procedures identical to those described for the initial 25 training trials in Experiment $I$. The four groups were then given 25 additional training trials (retraining) either $0,1,4$, or $24 \mathrm{~h}$ after original training. With the exception of the 0 -h group, which received 50 continuous training trials, the groups were returned to their home cages for the intertraining interval.

\section{Results}

Original training. There were no significant differences between the groups for either the escape latency or avoidance measure on original training (mdn avoidance responses $=5.5 ; \mathrm{mdn}$ escape latencies $=8.73 \mathrm{sec}$ ).

Retraining. The avoidance and escape latency data are summarized in Figure 2 as a function of time between original training and retraining. The time-dependent U-shaped function that defines the Kamin effect is clearly evident in the avoidance measure. The group retrained $1 \mathrm{~h}$ after original training performed fewer avoidance responses than did the $0-, 4-$, and 24 -h groups. The escape latency data also indicate a time-dependent change in performance. This is evident as an inverted U-shaped function with the escape latencies of the 1 -h group longer than the latencies of the $0-, 4$-, or 24-h groups.

Kruskall-Wallis analyses of variance demonstrated a significant change in both avoidance responses and escape latencies as a function of the interval between original training and retraining $(p<.05$ for both measures). Mann-Whitney $U$ tests (two-tailed) indicated that the $0-, 4-$, and $24-h$ groups did not differ 
Figure 2. Median avoidance responses and median of mean escape latencies as a function of the interval between original training and retraining. The avoidance measure for retraining is represented by open triangles and solid lines, and the escape measure is represented by open circles and broken lines. The avoidance measure for original training (combined groups) is represented by the shaded triangle, and the escape measure is represented by the shaded circle.

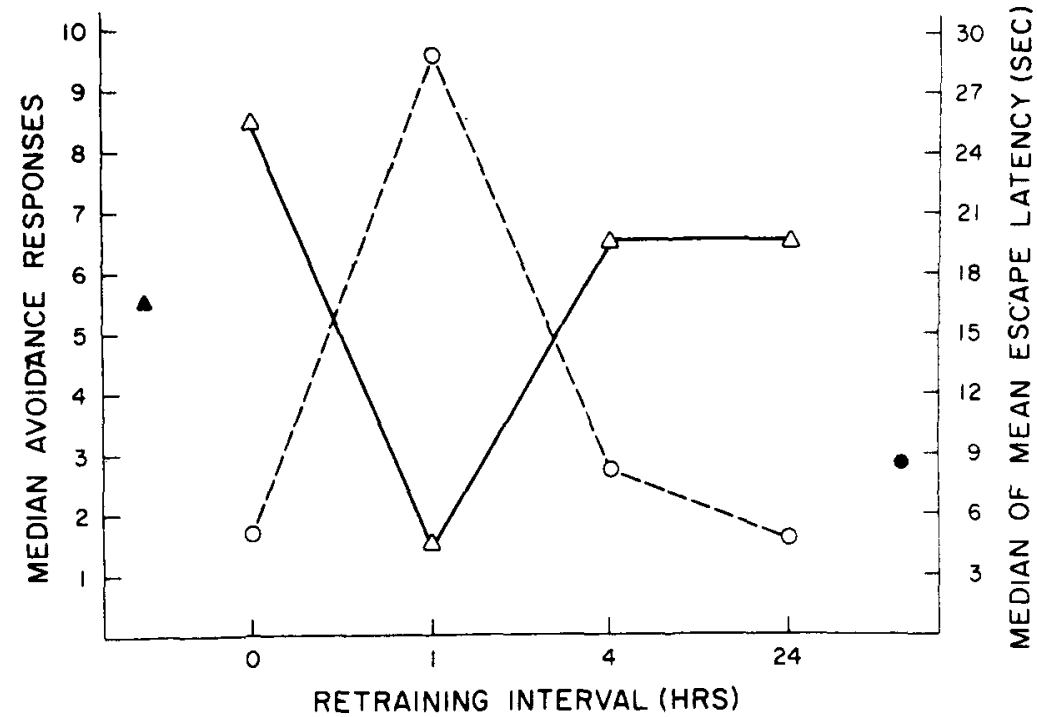

significantly from each other on number of avoidance responses or escape latencies. In contrast, the 1-h group performed significantly fewer avoidance responses than the 0 - and 4-h groups $(p<.04)$ and the 24 -h group $(p<.05)$. Escape latencies for the 1 h group were significantly longer than escape latencies for the $0 \cdot h$ $(p<.03)$ and $24-h(p<.04)$ groups but not the 4-h group. The magnitude of these performance changes was evaluated further by comparing performance on original training with performance on retraining for each group.

Original training and retraining. The performance of the $0-, 4-$, and $24-h$ groups tended to improve from original training to retraining, whereas performance of the $1 \mathrm{~h}$ group tended to deteriorate. The improved performance of the 0-, 4-, and 24-h groups was evident as an increase in number of avoidance responses $(\mathrm{mdn}=5.5$ on original training to 8.5 on retraining for the combined group data) and a decrease in escape latencies $(\mathrm{mdn}=8.65$ on original training to 5.07 on retraining). These performance changes, however, were not statistically significant (Wilcoxin matched-pairs signed-ranks test). For the 1-h group, deterioration in performance was evident as a decrease in number of avoidance responses $(\mathrm{mdn}=5.0$ on original training to 1.5 on retraining) and an increase in escape latencies ( $\mathrm{mdn}=8.89$ on original training to 29.01 on retraining). The change in escape latencies was significant $(p<.02)$, but the change in avoidance responses was not $(p<.08>.05)$.

\section{GENERAL DISCUSSION}

The data reported here clearly demonstrate the effectiveness of intense white noise as a UCS for shuttlebox avoidance conditioning. The results of Experiment I, together with previously reported results using intense noise as a UCS (Barnes \& Kish, 1957; Barry
\& Harrison, 1957; Bolles \& Seelbach, 1964; Campbell, 1955; Harrison \& Abelson, 1959; Harrison \& Tracy, 1955 ) suggest that the aversive properties of noise and shock are similar in that, for both stimuli, there appears to be an inverse relationship between the ease of conditioning a response and the complexity of the response to be conditioned. Whether or not this similarity reflects an SSDR (Bolles, 1971) common to noise and to shock cannot be determined from the data reported here. Further, while the results of Experiment I do not bear on whether or not intense noise is inherently less aversive than shock, the data are clearly inconsistent with the conclusion that noise is of little use in behavioral research (Campbell \& Bloom, 1965).

The results of Experiment II are in agreement with many previous reports demonstrating the Kamin effect with shock as the UCS. These data extend the generality of the ubiquitous Kamin effect. A unique contribution of the data reported here is that the Kamin effect is evident as an inverted U-shaped function in the escape latency measure. No experiments have reported performance changes related to responses made in the presence of the UCS in a Kamin effect paradigm. The escape latency data reported in Experiment II provide a measure of such performance changes and bear on theoretical interpretations of the underlying basis of the Kamin effect. The Kamin effect has been suggested to reflect transient stress-induced changes in memory or motivational processes. The diverse hypotheses that characterize these interpretations have been summarized elsewhere (Anisman, 1973; Barrett, Leith, \& Ray, 1971; Brush, 1971; Steranka \& Barrett, 1973). The fact that escape latencies for the $1 \mathrm{~h}$ group in Experiment II were reliably longer on retraining than on original training is not consistent with a memory interpretation of the Kamin effect (Grossman, 1967; Klein \& Spear, 1970; Spear, Klein, \& Riley, 1971). Such an interpretation 
predicts either no change from original training to retraining $1 \mathrm{~h}$ later, due to a complete retrieval failure, or slightly improved performance due to partial retrieval from original training.

The escape latency data from Experiment II are consistent with a motivational interpretation of the Kamin effect. The variety of motivational hypotheses offered to explain the Kamin effect include a transient increase in fear (Denny \& Ditchman, 1962; Kumar, 1970; McMichael, 1966; Tarpy, 1966), a transient decrease in fear (Bintz, 1970; Bintz, Braud, \& Brown, 1970; Pinel \& Cooper, 1966), and changes in central physiological mechanisms related to changes in activity (Anisman, 1973; Barrett, Leith, \& Ray, 1971; Carlton, 1969; Steranka \& Barrett, 1973). The avoidance data from Experiment II do not permit a choice between these alternative hypotheses. However, the escape latency data seem less clearly related to changes in fear than to changes in activity. The increased latencies in the 1 -h group were in response to presentations of the aversive UCS. Fear is a construct that is typically inferred from responses to stimuli that are paired with an aversive UCS. Further, whether or not fear in fact changes as a function of time after stress remains a controversial issue (McAllister \& McAllister, 1967). Thus, it would not appear particularly parsimonious to attribute the changes in escape latencies in Experiment II to changes in fear. Rather, the long escape latencies of the 1-h group in Experiment II may be most parsimoniously explained as due to a transient decrease in activity. Thus, these data contributed to an increasingly convincing body of data which suggests that the Kamin effect reflects a transient diminution in an animal's ability to perform an active response following stress (Anisman, 1973; Barrett, Leith, \& Ray, 1971; Carlton, 1969; Manto, 1967; Pinel \& Mucha, 1973; Sterenka \& Barrett, 1973).

\section{REFERENCES}

Anisman, $H$. Cholinergic mechanisms and alterations in behavioral suppression as factors producing time-dependent changes in avoidance performance. Journal of Comparative and Phy siological Psychology, 1973, 83, 465-477.

Bames, G. W., \& Kish, B. G. Reinforcing properties of the termination of intense auditory stimulation. Joumal of Comparative and Phy siological Psychology, 1957, 50, 40-43.

Barrett, R. J., Leith, N. J., \& Ray, O. S. Kamin effect in rats: Index of memory or shock induced inhibition. Joumal of Comparative and Physiological Psychology, 1971, 77, 234-239.

Barry, J. J., \& Harrison, J. M. Relation between stimulus intensity and strength of escape responding. Psychological Reports, 1957, 3, 3-8.
Bintz, J. Time-dependent memory deficits of aversively motivated behavior. Learning and Motivation, 1970, 1 , 405-406.

Bintz, J., Braud, W. G., \& Brown, J.S. An analy sis of the role of fear in the Kamin effect. Leaming and Motivation, 1970, 1, 170-176.

Bolles, R. C. Species-specific defense reactions. In F. R. Brush (Ed.), Aversive conditioning and learning. New York: Academic Press, 1971.

Bolles, R. C., \& Seelbach, S. E. Punishing and reinforcing effects of noise onset and termination for different responses. Journal of Comparative and Physiological Psychology, 1964, 58, 127-131.

Brush, F. R. Retention of aversively motivated behavior. In F. R. Brush (Ed.), Aversive conditioning and learning. New York: Academic Press, 1971.

Campbell, B. A. The fractional reduction in noxious stimulation required to produce "just noticeable", learning. Journal of Comparative and Physiological Psy chology, 1955, 50, 40.43.

Campbell, B. A., \& Bloom, J. M. Relative aversiveness of noise and shock. Journal of Comparative and Physiological Psychology, 1965, 60, 440-442.

Carlton, P. L. Brain acety lcholine and inhibition. In J. T. Tapp (Ed.), Reinforcement: Current research and theories. New York: Academic Press, 1969

Denny, M. R., \& Ditchman, R. E. The locus of maximal "Kamin effect" in rats. Journal of Comparative and Physiological Psy chology, 1962, 55, 1069-1070.

Grossman, S. P. A textbook of physiological psychology. New York: Wiley, 1967 .

Harrison, J. M., Abelson, R. M. The maintenance of behavior by the termination and onset of intense noise. Journal of Experimental Analysis of Behavior, 1959, 2, 23-42.

Harrison, J. M.. \& Tracy, W. H. The use of auditory stimuli to maintain lever pressing behavior. Science, 1955, 121, 373-374.

Kamin, L. J. Retention of an incompletely leamed avoidance response. Journal of Comparative and Physiological Psy chology, 1957, 50, 457-460.

Klein, S. B., \& Spear, N. E. Forgetting by the rat after intermediate intervals ("Kamin effect") as retrieval failure. Joumal of Comparative and Phy siological Psychology, 1970, 71, 165-170.

Kumar, R. Incubation of fear: Experiments on the "Kamin effect" in rats. Joumal of Comparative and Physiological Psychology, 1970, 70, 258-263.

Manto, P. G. Blockage of epinephrine induced decrement in activity by scopolamine. Psychonomic Science, 1967, 1, 203-204.

McAllister, D. E., \& McAllister, W, R. Incubation of fear: An examination of the concept. Joumal of Experimental Research in Personality, 1967, 2, 180-190.

McMichael, J. S. Incubation of anxiety and instrumental behavior. Joumal of Comparative and Physiological Psy chology, 1966, 61, 208-211.

Pinel, J. P. J., \& Cooper, R. M. Demonstration of the Kamin effect after one-trial avoidance learning. Psy chonomic Science, $1966,4,17-18$

Pinel, J. P. J., \& Mucha, R. F. Incubation and Kamin effects in the rat: Changes in activity and reactivity after footshock. Joumal of Comparative and Phy siological Psychology, 1973, 84, 661-668.

Seigel. S. Nonparametric statistics for the behavioral sciences. New York: McGraw-Hill, 1956.

Spear, N. E., Klein, S. B., \& Riley, E. P. The Kamin effect as "state-dependent learning": Memory-retrieval failure in the rat. Joumal of Comparative and Physiological Psychology, $1971,74,416-425$.

Steranka, L. R., \& Barrett, R. J. Kamin effect in rats: Differential retention or differential acquisition of an active avoidance response. Journal of Comparative and Phy siological Psy chology, 1973, 85, 324-330.

Tarpy, $R$. Incubation of anxiety as measured by response suppression. Psy chonmic Science, 1966, 4, 189-190.

(Received for publication November 14,1974; revision accepted March 12, 1975.) 